\title{
Symptom Status Predicts Patient Outcomes in Persons with HIV and Comorbid Liver Disease
}

\author{
Wendy A. Henderson, ${ }^{1}$ Angela C. Martino, ${ }^{1}$ Noriko Kitamura, ${ }^{1}$ \\ Kevin H. Kim, ${ }^{2}$ and Judith A. Erlen ${ }^{3}$ \\ ${ }^{1}$ Biobehavioral Unit, National Institute of Nursing Research, National Institutes of Health, DHHS, 10 Center Drive, \\ Room 2-1339, Bethesda, MD 20892, USA \\ ${ }^{2}$ School of Education, University of Pittsburgh, 5918 Wesley W. Posvar Hall, 230 South Bouquet Street, Pittsburgh, \\ PA 15261, USA \\ ${ }^{3}$ Department of Health and Community Systems, School of Nursing, University of Pittsburgh, 415 Victoria Building, \\ 3500 Victoria Street, Pittsburgh, PA 15261, USA
}

Correspondence should be addressed to Wendy A. Henderson, hendersw@mail.nih.gov

Received 1 June 2012; Revised 6 September 2012; Accepted 7 September 2012

Academic Editor: Xia Jin

Copyright ( $) 2012$ Wendy A. Henderson et al. This is an open access article distributed under the Creative Commons Attribution License, which permits unrestricted use, distribution, and reproduction in any medium, provided the original work is properly cited.

Persons living with human immunodeficiency virus (HIV) are living longer; therefore, they are more likely to suffer significant morbidity due to potentially treatable liver diseases. Clinical evidence suggests that the growing number of individuals living with HIV and liver disease may have a poorer health-related quality of life (HRQOL) than persons living with HIV who do not have comorbid liver disease. Thus, this study examined the multiple components of HRQOL by testing Wilson and Cleary's model in a sample of 532 individuals (305 persons with HIV and 227 persons living with HIV and liver disease) using structural equation modeling. The model components include biological/physiological factors (HIV viral load, CD4 counts), symptom status (Beck Depression Inventory II and the Medical Outcomes Study HIV Health Survey (MOS-HIV) mental function), functional status (missed appointments and MOS-HIV physical function), general health perceptions (perceived burden visual analogue scale and MOS-HIV health transition), and overall quality of life (QOL) (Satisfaction with Life Scale and MOS-HIV overall QOL). The Wilson and Cleary model was found to be useful in linking clinical indicators to patient-related outcomes. The findings provide the foundation for development and future testing of targeted biobehavioral nursing interventions to improve HRQOL in persons living with HIV and liver disease.

\section{Introduction}

Since the introduction of highly active antiretroviral therapy (HAART), survival of persons living with human immunodeficiency virus (HIV) has dramatically improved. Five-year survival rate among patients following World Health Organization (WHO) standard therapies reaches approximately $75 \%$ [1]. The result is that persons living with HIV are more likely to suffer significant morbidity and mortality from other disorders such as liver disease (LD) and its related complications (anemia, end stage liver disease, lipodystrophy, and hepatocellular carcinoma) than from HIV [2]. Because of the toxic and metabolic effects of antiretroviral medications on the liver and coinfection with
LD, the number of persons living with HIV and LD is increasing [3-6].

Hepatitis $\mathrm{B}(\mathrm{HBV})$ and $\mathrm{C}$ virus $(\mathrm{HCV})$ infections are prevalent among HIV-infected individuals with different epidemiologic profiles, modes of transmission, natural histories, and treatments [7]. It is estimated that 3 to 6 million people are infected with HIV and chronic HBV worldwide, which is approximately $10 \%$ of HIV-positive persons. In the USA, where HBV and HIV are most often acquired by sexual transmission or injection-drug use and $\mathrm{HBV}$ prevalence is low, the prevalence of HIV-HBV coinfection in HIVpositive population is generally less than $10 \%$. However, the prevalence is up to $50 \%$ among injection-drug users with HIV [8]. Generally, one-third of persons with HIV also have 
chronic HCV infection. In the USA, approximately 300000 individuals are living with HIV coinfected chronic HCV [9]. HIV and HCV coinfection increases up to $70-90 \%$ of hemophilia and $60-80 \%$ of injection-drug users who have high risk of blood exposure [10].

In addition, HIV infection accelerates the natural course of HBV and HCV infections, including death, histological fibrosis/cirrhosis, decompensated liver disease, and hepatocellular carcinoma [11-13]. More recently, the evidence shows that HIV itself and immunosuppression contributes to the liver injury. Also, antiretroviral therapy (ART) attenuates the progress of HBV and HCV [12]. Furthermore, among certain populations, such as the homeless or incarcerated individuals with HIV, the prevalence of liver disease reaches $69 \%$ [14]. Nonalcoholic steatohepatitis (NASH) is also increasing in HIV-positive populations [12]. A recent study showed that fatty acid production increases in HIV and HCV coinfection [15].

Treatment advances have improved survival rates for HIV-infected individuals, although not always with a good quality of life (QOL). Deteriorated liver conditions have shown to have a significant negative effect on persons' healthrelated quality of life (HRQOL) [16]. In addition, HIVpositive individuals with liver disease are likely from socially vulnerable groups, such as injection-drug users, the homeless, and incarcerated people. Therefore, it is important to identify interventions that have the potential to improve QOL in people living with HIV and liver diseases (LD). An overarching goal of Healthy People 2020 is to increase life expectancy and promote QOL of individuals of all ages [17]; the challenge for researchers and practitioners is to first determine what aspects of individuals' HRQOL are affected when they live with multiple comorbid conditions.

One framework for examining HRQOL is the model proposed by Wilson and Cleary (1995) [18]. The primary aim was to test the null hypothesis, wherein, the hypothesized model would hold true with the components (biologi$\mathrm{cal} /$ physiological factors, symptom status, functional status, general health perceptions, and overall quality of life) in persons living with HIV without LD and in persons living with HIV and LD. The null hypothesis was that there would be no difference in the model in persons living with HIV without LD and in persons living with HIV and LD. The secondary aim of the study was to test the relationships proposed within Wilson and Cleary's directional model of HRQOL between biological/physiological factors, symptom status, functional status, general health perceptions, and overall quality of life among persons living with HIV without LD and persons living with HIV and LD. The knowledge gained from persons with HIV and LD in this study may provide a way to support people living with this complex illness.

\section{Materials and Methods}

2.1. Participants. HIV-positive individuals who were currently treated with antiretroviral drugs, at least 18 years of age, and had telephone access were included in the study. All participants gave written informed consent.

2.2. Study Design and Data Collection. The parent study was a randomized controlled trial supported by the National Institute of Nursing Research testing interventions to improve medication adherence in persons living with HIV. Participant data was collected between April 1999 and March 2007 from western Pennsylvania and eastern Ohio community hospitals, university-based clinics, and comprehensive HIV care centers. A history of HIV and LD was ascertained by self-reported data from the Comorbidity Questionnaire, Center for Research in Chronic Disorders (CRCD), University of Pittsburgh School of Nursing (1999), and medical record review. The type of $\mathrm{LD}$ was confirmed through medical record review by a registered nurse and from selfreport. LD included but was not limited to hepatitis (A, $\mathrm{B}, \mathrm{C}$, and other), cirrhosis, steatosis, and hepatocellular carcinoma. The precise nature of $\mathrm{LD}$ was not available in 5\% of cases.

2.2.1. Wilson and Cleary Health-Related Quality of Life Framework. HRQOL was defined as the state of perceived health and its effect on the person [18-25]. The Wilson and Cleary model of HRQOL includes health-related and social factors. As a causal model, Wilson and Cleary allows for identification of potential causal factors in the overall HRQOL paradigm.

Each of the components in the Wilson and Cleary model (biological/physiological factors, symptom status, functional status, general health perceptions, and overall QOL) has been addressed separately in numerous studies. The researchers reviewed those studies that were relevant to HIV and liver disease and to the operationalized Wilson and Cleary model of HRQOL (Figure 1). The definition of each parameter of Wilson and Cleary's model is described below.

Biological/Physiological Factors. Biological/physiological factors are any measurable function of the cells or organs of an individual. This component includes other clinical indicators such as measures of change in the function of the cell, organ, or organ system. In this study, HIV viral load and CD4 counts were used as biological and physiological factors that were assessed.

Symptom Status. Symptom status was described theoretically as any psychophysical, emotional, or cognitive state that influences the individual [18]. Often depressive symptoms and mental health are included in this definition. Symptom status was operationally assessed using each subject's Beck Depression Inventory II (BDI-II) score and the Medical Outcomes Study HIV Health Survey (MOS-HIV) mental function summary score [26].

Functional Status. Functional status was defined as one's ability to perform specific tasks such as going to work or making and keeping medical appointments [18]. Functional status was measured with the MOS-HIV physical function 


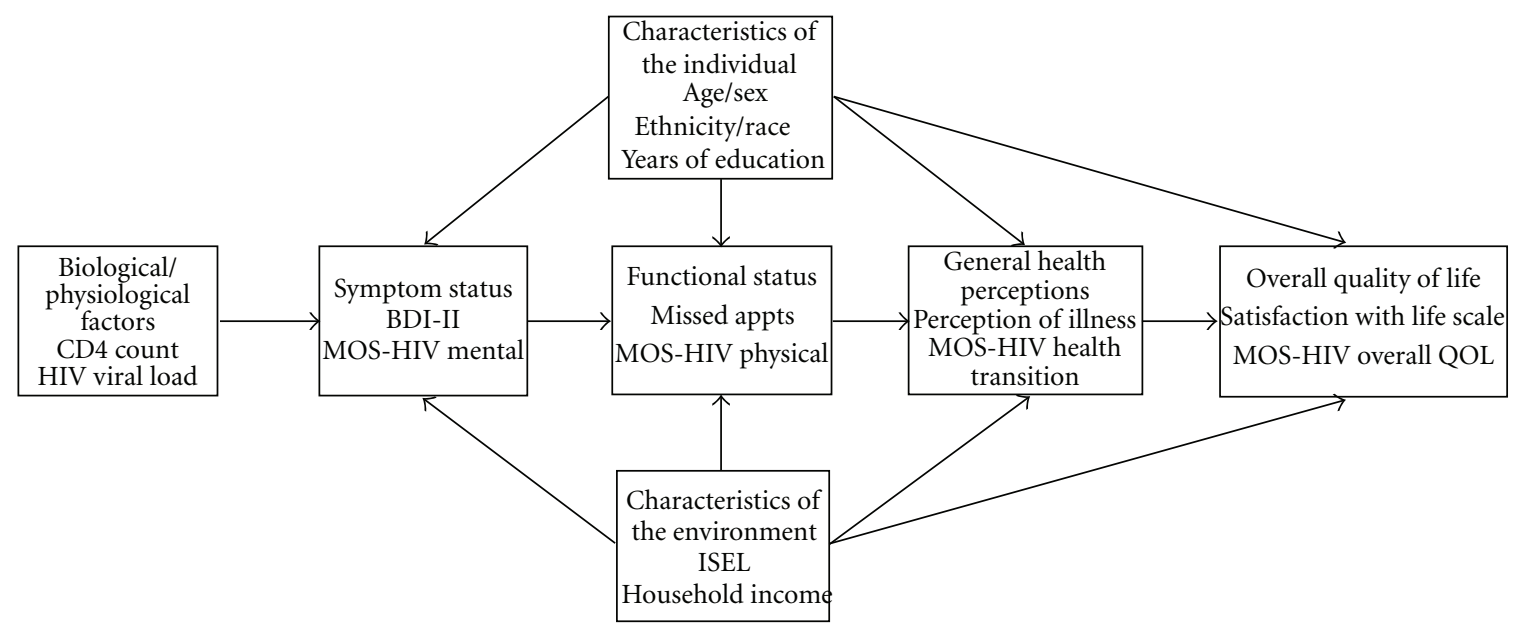

Figure 1: Operationalized Wilson and Cleary model, modified from [18].

summary score [26] and missed clinic appointments during the prior 6 months. The MOS-HIV physical function summary score was used to report self-perceived functionality [26]. Missed clinic appointments were quantified using selfreport or chart review data from the medical record review and were categorized as missed or not missed.

General Health Perceptions. General health perceptions were theoretically defined as how individuals perceive their own health, based on the integration of biological/physiologic factors, symptom status, and functional status combined with the effect of the particular disease or organ state on the individual [18]. General health perceptions were measured by the Perception of Illness Visual Analog Scale [27] and the single item MOS-HIV health transition score [26].

Overall Quality of Life. Theoretically, overall quality of life was described as how satisfied individuals are with all aspects of their life [18]. Overall quality of life was measured with the Satisfaction with Life Scale [28] and a single MOS-HIV item assessing overall HRQOL [26].

Characteristics of the Individual. Characteristics of the individual were specific descriptors of the person [18]. In this study, age (measured in years), sex (categorized as male or female), ethnicity/race (categorized as white and nonwhite and further categorized based on self-report), and number of years of education were included. This demographic information was collected using the CRCD Socio-demographic Questionnaire, University of Pittsburgh, School of Nursing (1999) or via medical record review.

Characteristics of the Environment. The characteristics of the environment theoretically incorporated all of the individual's surroundings, including tangible or intangible available resources [18]. An intangible resource that is often associated with health outcomes is social support. Social support was limited to one measure of subjective and one measure of objective social support. The subjective measure of the extent to which individuals feel that they have interpersonal resources available to them was ascertained from the total score of the Interpersonal Support Evaluation List (ISEL) [29]. Income, as a potentially tangible supportive influence from an individual's environment, was measured by annual gross household income.

2.3. Data Analysis. Data were compiled from the baseline data collection and medical record review from the parent study. All persons living with HIV who self-reported a "liver problem" were included in the HIV and LD group regardless of available objective medical record data. All persons living with HIV who had an LD noted in their baseline medical record were also included in the LD group. All others were considered persons living with HIV without LD.

Each variable was examined for its distribution, range, mean, median, mode, and standard deviation. Assessment was done to test for normality specific to the type of variable. For dichotomous variables, frequencies were explored to identify whether cell sizes were relatively equal. Chi-square and the independent samples $t$-test were used to examine differences between the initial and continuation studies and the two groups: persons living with HIV and LD and persons living with HIV without LD. Correlations between the variables were analyzed for systematic entry into the structured equation model (SEM). SPSS version 15.0 (SPSS Inc., Chicago, IL, USA) was used to further manage and analyze the data.

A multigroup structural equation modeling (SEM) was used to test the fit of the Wilson and Cleary (1995) model of HRQOL in the two groups. A maximum likelihood estimation method used EQS software package version 6.1 (Multivariate Software, Inc., Encino, CA, USA) to perform the statistical analyses [30].

In addition, multiple pathways were added to SEM model to identify whether there were different relationships between characteristics of individuals and environment between and among persons living with HIV without liver disease and persons living with HIV and LD. Model paths 


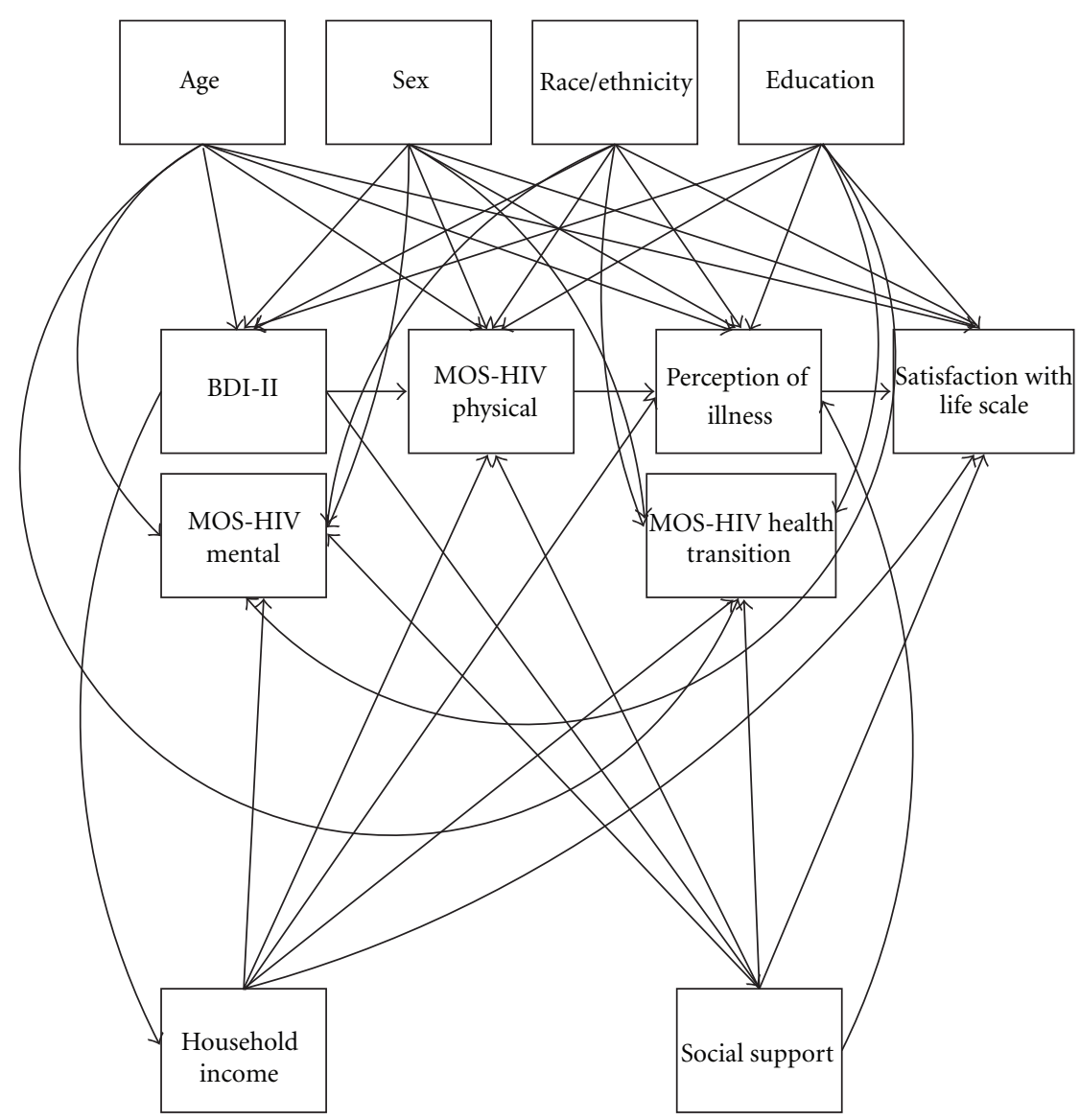

Figure 2: Additional 36 covariate relationships tested in exploratory SEM.

included: paths from each of the four selected demographic characteristics to the four endogenous variables (symptom status, functional status, general health perceptions, and overall QOL) (16 paths); correlations among the four selected demographic characteristics (6 paths) and paths from each of the four endogenous variables to the two characteristics of the environment (household income and social support), along with the correlation between those two characteristics ( 9 paths). These paths are depicted graphically in Figure 2.

Age, gender, ethnicity/race, and number of years of education were included as characteristics of the individual and social support (measured with the mean score on the Inventory Support Evaluation List (ISEL)), and household income (measured as total gross annual household income) as characteristics of the environment.

For determination of adequacy of sample size the observation per parameters (N:q) was calculated. Generally, if more than five observations are present per parameter the sample size for the model is deemed adequate. In this study, the number of observations (sample size) was 532 and the number of the largest parameters included (covariates in the model) was 36 . Therefore, N:q for model testing was 14.8 , which is greater than five. Thus, the sample size was deemed sufficient for the model [31].

\section{Results}

A total of 532 individuals living with HIV (305 with HIV and 227 with HIV and LD) were included in the study. There were no significant differences between the groups with regard to gender, race, employment status, and household income. However, subjects with HIV and LD were significantly older and less educated than the HIV group without LD (Table 1). Participants had a mean CD4 count of 455 cells $/ \mathrm{mm}^{3}$ (range 44-1540 cells $/ \mathrm{mm}^{3}$ ) and $59 \%$ of the overall sample had an undetectable HIV viral load (Table 2). The classifications of types of comorbid LD are noted in Table 3. All others without evidence of LD were classified as HIV.

The baseline hypothesized Wilson and Cleary model was assessed with SEM and found to hold true in both groups, persons living with HIV without LD and in persons living with HIV and LD, when a Lagrange multiplier modification was applied. The baseline model parameters and subsequent model modifications are noted in Table 4 . Model 1 parameters were found after releasing model constraints and allowing additional pathways from symptom status to general health perceptions and overall quality of life, and from biological/physiological factors to general health perceptions. Model 2 allowed for correlations between the biological/ physiological factors (CD4 count and HIV viral load) and 
TABLE 1: Characteristics of demographics in HIV and HIV + LD groups.

\begin{tabular}{|c|c|c|c|c|c|c|}
\hline \multirow{4}{*}{ Variable } & \multirow{4}{*}{$\begin{array}{l}\text { Overall }(N=532) \\
n(\%) / M(\mathrm{SD})\end{array}$} & \multicolumn{2}{|c|}{ Group } & \multicolumn{3}{|c|}{ Statistic } \\
\hline & & \multirow{3}{*}{$\begin{array}{c}\operatorname{HIV}(N=305) \\
n(\%) / M(\mathrm{SD})\end{array}$} & \multirow{3}{*}{$\begin{array}{c}\mathrm{HIV}+\mathrm{LD}(N=227) \\
n(\%) / M(\mathrm{SD})\end{array}$} & \multirow{2}{*}{\multicolumn{2}{|c|}{ Chi-sq/t-test }} & \multirow{3}{*}{$P$ value } \\
\hline & & & & & & \\
\hline & & & & $d f$ & Value & \\
\hline Sex & & & & 1 & 1.63 & .201 \\
\hline Male & $371(69.7)$ & $206(67.5)$ & $165(72.7)$ & & & \\
\hline Female & $161(30.3)$ & $99(32.5)$ & $62(27.3)$ & & & \\
\hline Race & & & & 1 & 1.79 & .181 \\
\hline White & $261(49.1)$ & $142(46.6)$ & $119(52.4)$ & & & \\
\hline Non-white & $271(50.9)$ & $163(53.4)$ & $108(47.6)$ & & & \\
\hline Total gross annual household income & & & & 5 & 8.08 & .152 \\
\hline Under 10000 & $262(49.2)$ & $140(45.9)$ & $122(52.9)$ & & & \\
\hline 10000 to 13000 & $90(16.9)$ & $53(17.4)$ & $37(16.3)$ & & & \\
\hline 13000 to 20000 & $60(11.3)$ & $35(11.5)$ & $25(11.0)$ & & & \\
\hline 20000 to 30000 & $39(7.3)$ & $29(9.5)$ & $10(4.4)$ & & & \\
\hline 30000 to 50000 & $34(6.4)$ & $23(7.5)$ & $11(4.8)$ & & & \\
\hline Over 50000 & $32(6.0)$ & $17(5.6)$ & $15(6.6)$ & & & \\
\hline Missing & $15(2.8)$ & $8(2.6)$ & $7(3.1)$ & & & \\
\hline Age & $42.40(7.86)$ & $41.51(8.29)$ & $43.87(7.14)$ & 530 & -3.44 & .001 \\
\hline Number of years of education & $13.21(2.76)$ & $13.35(2.83)$ & $12.77(2.66)$ & 529 & 2.37 & .018 \\
\hline
\end{tabular}

TABLE 2: Measure comparisons by model variable for HIV and HIV + LD groups.

\begin{tabular}{|c|c|c|c|c|c|c|}
\hline \multirow{4}{*}{ Variable measure } & \multirow{4}{*}{$\begin{array}{l}\text { Overall }(n=532) \\
n(\%) / M(\mathrm{SD})\end{array}$} & \multicolumn{2}{|c|}{ Group } & \multicolumn{3}{|c|}{ Statistic } \\
\hline & & \multirow{3}{*}{$\begin{array}{l}\operatorname{HIV}(n=305) \\
n(\%) / M(\mathrm{SD})\end{array}$} & \multirow{3}{*}{$\begin{array}{c}\mathrm{HIV}+\mathrm{LD}(n=227) \\
n(\%) / M(\mathrm{SD})\end{array}$} & \multirow{2}{*}{\multicolumn{3}{|c|}{ Chi-sq/t-test }} \\
\hline & & & & & & \\
\hline & & & & $d f$ & Value & $P$ value \\
\hline \multicolumn{7}{|l|}{ Biological/physiological factors } \\
\hline CD4 count & $455.94(303.97)$ & $492.25(341.98)$ & $435.43(316.30)$ & Mann-Whitney & -2.01 & .044 \\
\hline HIV viral load & & & & Pearson (1) & 3.35 & .067 \\
\hline Detectable & $199(41.1)$ & $102(37.5)$ & $97(45.8)$ & & & \\
\hline Undetectable & $285(58.9)$ & $170(62.5)$ & $115(54.2)$ & & & \\
\hline \multicolumn{7}{|l|}{ Symptom status } \\
\hline Beck depression inventory-II & $14.94(11.54)$ & $13.89(11.68)$ & $16.34(11.22)$ & 525 & -2.42 & .016 \\
\hline MOS-HIV mental summary score & $45.44(12.06)$ & $46.77(12.24)$ & $43.63(11.59)$ & 520 & 2.96 & .003 \\
\hline \multicolumn{7}{|l|}{ Functional status } \\
\hline Missed appointments & & & & Pearson (1) & .835 & .361 \\
\hline Yes & $172(33.0)$ & $94(31.3)$ & $78(35.1)$ & & & \\
\hline No & $350(67.0)$ & $206(68.7)$ & $144(64.9)$ & & & \\
\hline MOS-HIV physical summary score & $41.80(11.62)$ & $43.47(11.28)$ & $39.51(11.72)$ & 520 & 3.90 & $<.001$ \\
\hline Perception of illness & $0.74(0.19)$ & $.74(.18)$ & $.731(.19)$ & 524 & .72 & .470 \\
\hline MOS-HIV health transition score & $3.34(1.02)$ & $3.39(1.01)$ & $3.27(1.02)$ & 524 & 1.34 & .182 \\
\hline \multicolumn{7}{|l|}{ Overall quality of life } \\
\hline Satisfaction with life scale & 3.57 (1.49) & $3.63(1.44)$ & $3.49(1.56)$ & 517 & 1.08 & .280 \\
\hline
\end{tabular}

the error terms. There were no significant differences in the HIV group model and the HIV and LD model. A multisample robust SEM was performed. Because there were no significant differences between the structural models in the two groups the data were constrained to fit the same model. The constrained model estimates one set of parameters for both groups. SEM was run on both the constrained and unconstrained models and it was found that the constrained model had a better fit. The model parameters were not significantly different when comparing the Satorra-Bentler model chi-squares.

There was a significant direct effect of biological/ physiological factors on symptom status, as measured with the MOS-HIV mental summary score, in persons with HIV and persons with HIV and LD. CD4 count, as a measure of biological/physiological factors, significantly predicted 
TABLE 3: Classification of comorbid types of liver disease $(n=227)$.

\begin{tabular}{lccc}
\hline HIV and type of liver disease & $N$ & Cumulative total & Percentage (\%) \\
\hline HIV + Hepatitis A only & 15 & 15 & 6.6 \\
HIV + Hepatitis B only & 32 & 135 & 14.1 \\
HIV + Hepatitis C only & 88 & 142 & 38.8 \\
HIV + Hepatitis A and B & 7 & 143 & 3.1 \\
HIV + Hepatitis A and C & 1 & 156 & 0.4 \\
HIV + Hepatitis B and C & 13 & 161 & 5.7 \\
HIV + Hepatitis A, B and C & 5 & 213 & 2.2 \\
HIV + Unknown Hepatitis & 52 & 225 & 22.9 \\
HIV + Other liver disease & 12 & 226 & 5.3 \\
HIV + Other liver disease + Hepatitis C & 1 & 227 & 0.4 \\
HIV + Other liver disease + Hepatitis A, B, and C & 1 & 0.4 \\
\hline
\end{tabular}

TABLE 4: Goodness of fit summary for model selection.

\begin{tabular}{|c|c|c|c|c|c|c|c|}
\hline & $\mathrm{SB} \chi^{2}$ & $d f$ & CFI & RMSEA & $\Delta \mathrm{SB} \chi^{2}$ & $d f$ & $P$ value \\
\hline Baseline & 22.68 & 20 & .997 & .028 & - & - & - \\
\hline Model 1 regression coefficent constrained & 39.31 & 36 & .997 & .020 & 16.61 & 16 & .411 \\
\hline Model 2 regression coefficent constraints with parsimony & 40.31 & 38 & .998 & .017 & 17.57 & 18 & .484 \\
\hline
\end{tabular}

Note: SB $\chi^{2}$ : Satorra-Bentler scaled chi-square; $d f$ : degrees of freedom; CFI: comparative fit index; RMSEA: root mean squared error of approximation; $\Delta$ : difference.

symptom status, as measured with the MOS-HIV mental summary score in both the HIV $(B=.120, z=2.44, P=.015)$ and HIV and LD groups $(B=.113, z=2.44, P=.015)$. HIV viral load as a measure of biological/physiological factors significantly predicted symptom status, as measured with the MOS-HIV mental summary score in both the HIV $(B=.103$, $z=2.10, P=.036)$ and HIV and LD groups $(B=.107, z=$ $2.10, P=.036)$. An additional significant path was identified using the Lagrange multiplier from biological/physiological factors to general health perception (CD4 count to perception of illness) in both the HIV group $(B=.122, z=2.97, P=$ $.003)$ and the HIV and LD group $(B=.103, z=2.97, P=$ $.003)$. CD4 count and HIV viral load, as measures of biological/physiological factors, did not predict symptom status, as measured by the BDI-II.

There was a significant direct effect of symptom status on functional status in both groups. However, categorized missed appointments ("yes" or "no") was not related to the study measures and was therefore not included in the SEM/path analysis. The MOS-HIV mental summary score, as a measure of symptom status, significantly predicted functional status, as measured with the MOS-HIV physical summary score, in both the HIV $(B=.669, z=11.52, P<$ $.001)$ and HIV and LD groups $(B=.644, z=11.52, P<.001)$. The BDI-II, as a measure of symptom status, did not predict functional status, as measured by the MOS-HIV physical summary score.

There were four significant additional paths identified by using the Lagrange multiplier linking symptom status, as measured by the BDI-II and the MOS-HIV mental summary score, to distal components of the Wilson and Cleary model of HRQOL. Three of the identified paths linked symptom status directly to general health perceptions and one linked symptom status directly to overall QOL.
The MOS-HIV mental summary score, as a measure of symptom status, significantly predicted general health perceptions, as measured with the perception of illness visual analogue scale in both the $\operatorname{HIV}(B=.410, z=4.63, P<.001)$ and HIV and LD groups $(B=.369, z=4.63, P<.001)$. The MOS-HIV mental summary score, as a measure of symptom status, significantly predicted general health perceptions, as measured with the MOS-HIV health transition score, in both the HIV $(B=.343, z=4.12, P<.001)$ and HIV and LD groups $(B=.319, z=4.12, P<.001)$. The BDI-II, as a measure of symptom status, significantly predicted general health perceptions, as measured with the MOS-HIV health transition score in both the HIV $(B=-.204, z=-2.94, P=$ $.003)$ and HIV and LD groups $(B=-.203, z=-2.94$, $P=.003)$. The final added path included the BDI-II, as a measure of symptom status, which significantly predicted overall QOL, as measured with the satisfaction with life scale, in both the HIV $(B=.387, z=-6.84, P<.001)$ and HIV and LD groups $(B=.358, z=-6.84, P<.001)$.

There was no significant direct effect of functional status, as measured with the MOS-HIV physical summary score, on general health perceptions, as measured with either the perception of illness visual analogue score or the MOS-HIV health transition score, in persons with HIV without liver disease or in persons living with HIV and LD.

There was no significant direct effect of general health perceptions, as measured by either the perception of illness visual analogue score or the MOS-HIV health transition score, on overall QOL, as measured by the satisfaction with life scale, in either group. The significant retained SEM modeled pathways are depicted in Figure 3.

Of the 36 covariance pathways that were added to the good-fitting model, 11 individual parameters were found to be significant. There were four significant pathways found 


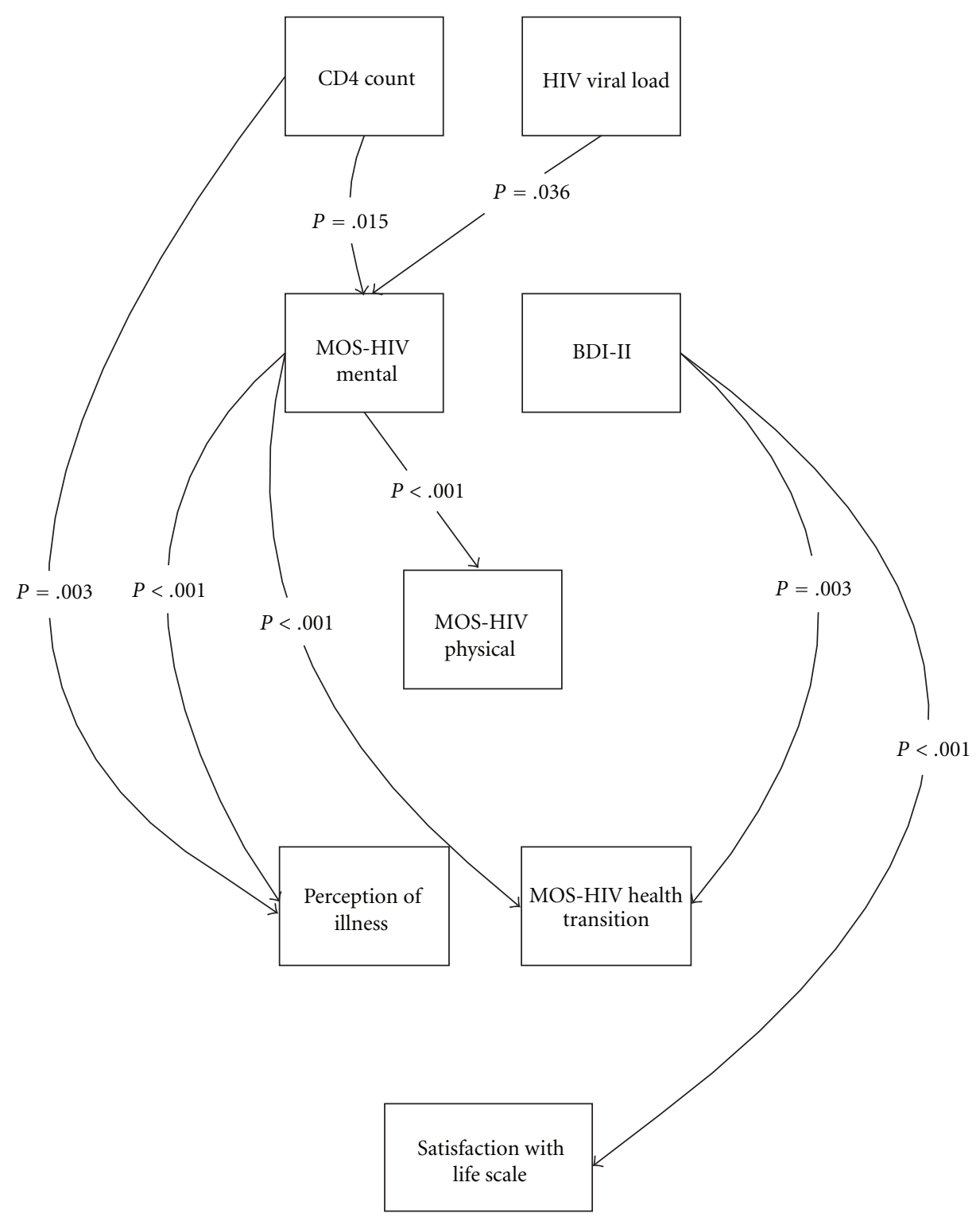

FIGURE 3: SEM with significant measured modeled pathways retained $(n=532)$.

stemming from the characteristics of the individual. Three of the four came from race/ethnicity and the remaining path related age to functional status. The covariate of age predicted the endogenous variable of functional status, as measured by the MOS-HIV physical summary score $(B=$ $.069, z=-1.984, P=.047)$. Age was not related to measures of symptom status, general health perceptions, or overall QOL. Race as a covariate in the model was found to have three independent significant paths. The first path that race predicted was symptom status, as measured by the MOSHIV mental summary score $(B=.091, z=2.204, P=.028)$. The second path predicted by race was functional status, as measured by the MOS-HIV physical summary score $(B=$ $.125, z=3.350, P=.001)$. The last path predicted by race was general health perceptions, as measured by the MOSHIV health transition score $(B=.148, z=3.443, P=$ $.001)$. Sex and years of education were not significantly related to the main Wilson and Cleary model components (biological/physiological factors, symptom status, functional status, general health perceptions, and overall QOL).

The characteristics of the environment as measured by the ISEL (social support) and household income had significant independent covariant relationships to the model parameters. Four paths linked from social support and three paths linked from gross annual household income. Specifically, self-reported social support, as measured with the ISEL, had an independent effect on symptom status at a significance level of $<.001$ as measured by both the BDI-II ( $B=$ .517, $z=-11.936, P<.001)$ and MOS-HIV mental summary score $(B=.525, z=11.685, P<.001)$. The ISEL score as a covariate in the model also independently predicted general health perceptions, as measured by the perception of illness visual analogue scale $(B=.228, z=4.180, P<$ $.001)$. Lastly, overall QOL, as measured by the satisfaction 


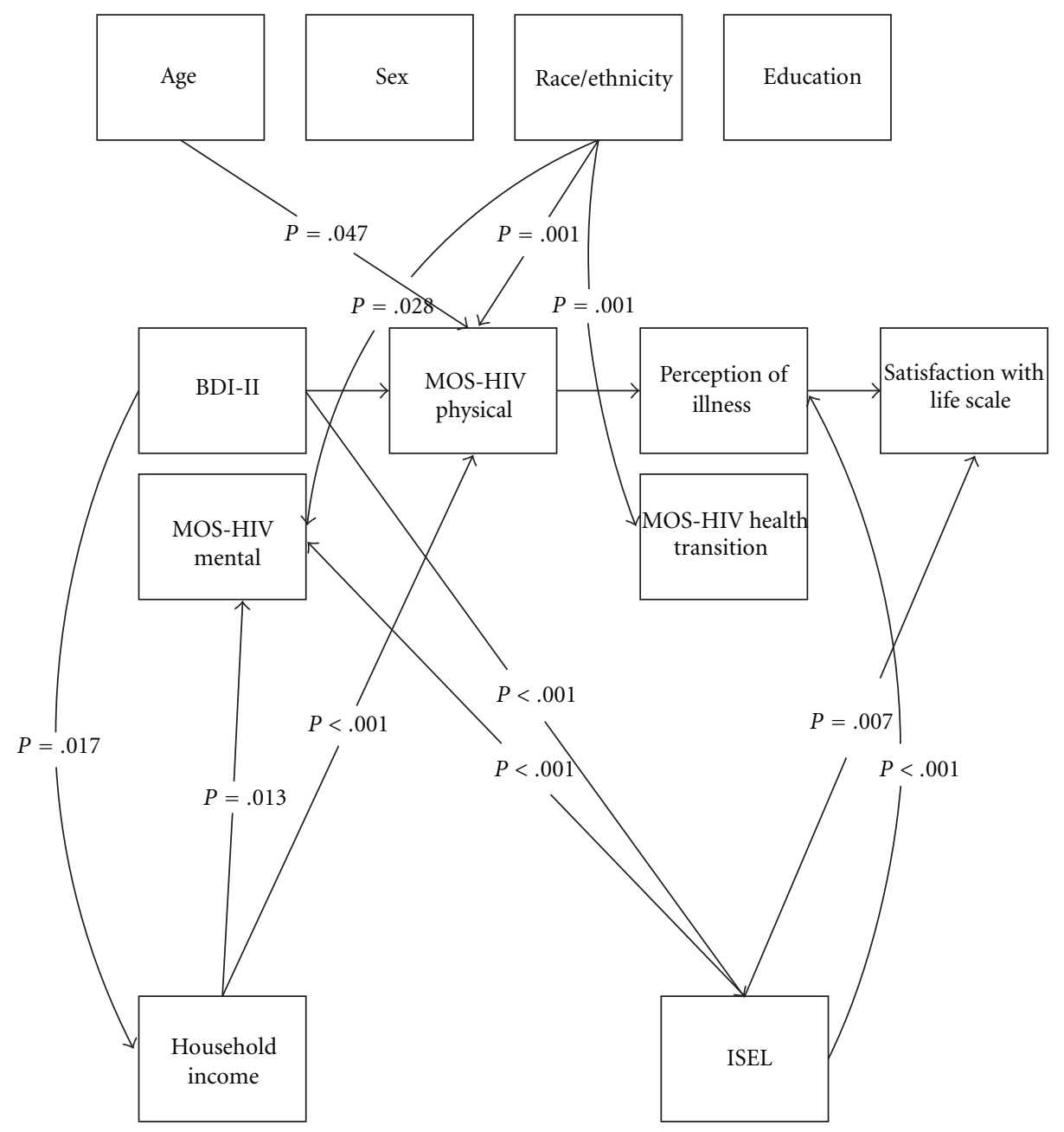

FIGURE 4: SEM with significant covariate model pathways retained.

with life scale, was independently predicted by the ISEL ( $B=$ $.155, z=2.707, P=.007)$. Gross annual household income, as a measure of characteristics of the environment, had three significant independent paths. First, gross annual household income as a covariate in the model predicted symptom status by both the BDI-II $(B=.104, z=-2.392, P=.017)$ and the MOS-HIV mental summary score $(B=.113, z=2.488, P=$ $.013)$. Gross annual household income also independently predicted functional status, as measured by the MOS-HIV physical summary score $(B=.165, z=4.024, P<.001)$. The significant retained independent multigroup SEM covariate pathways are depicted in Figure 4.

\section{Discussion}

This study applied a sophisticated statistical analysis, SEM, to test a theoretical model of HRQOL as described by Wilson and Cleary. The assessment of HRQOL is useful not only for capturing important facets of a person's self-perception of how illness affects daily functioning, but also as a valid measure of clinical outcome when assessing interventions. This model, as initially conceptualized by Wilson and Cleary, has been found useful to describe HRQOL in persons living with HIV. However, persons living with HIV have an increased risk of developing liver disease as related to toxic effects of antiretroviral therapy yielding hepatitis and other liver disease. These findings are similar to other studies that have used the Wilson and Cleary model in clinical samples with heart failure, gastrointestinal bleeding, diabetes, and Hodgkin's lymphoma. Therefore, the model proposed by Wilson and Cleary has now been supported in a sample of individuals with HIV and LD.

The primary aim of the study was to test the fit of the Wilson and Cleary model of HRQOL in two groups of patients, persons living with HIV without LD and persons living with HIV and LD. Wilson and Cleary's model was found to be fit for both groups.

The secondary aim was to test if each variable was directly linked as hypothesized by Wilson and Cleary. In both groups, the biological/physiologic factors (CD4 count and HIV viral load) had a significant direct effect on symptom status, which in turn had a significant direct effect on functional status. The additional pathways between symptom status and other components, such as QOL, have been noted by other researchers and suggest that self-reported depressive 
symptoms and mental function are important indicators of HRQOL [32-42].

Other possible factors influencing HRQOL, such as characteristics of the individual and of the environment, were investigated. Additionally, 36 parameters were assessed simultaneously. Social support was associated with both general health perceptions and overall QOL. Social support was also significantly related to mental symptom status. Total gross annual household income was related to MOS-HIV mental and physical summary scores. Thus, characteristics of the environment, as others have shown, have relevance to the components of HRQOL [43-45].

There are several limitations in this study. First, a longitudinal study would be necessary with data gathered from different time points to assess the causal relationships. The data used in this study were cross-sectional and therefore the causal relationships were not able to be assessed. Second, the higher proportion of non-white participants and lower income levels, or specific populations such as homeless and injection-drug users, may aid in making this study more generalizable as these differences are more reflective of the overall U.S. population of persons living with HIV. In addition, not having access to a telephone may have further biased the sample recruited.

\section{Conclusions}

The findings of this research show that both health-related factors, such as CD4 count and HIV viral load, and social factors, such as self-reported mental health and depressive symptoms, are important indicators of HRQOL in this sample of persons living with HIV $(N=532)$. These findings imply that symptom status, specifically depressive symptoms and altered mental function, is a key issue in determining HRQOL in persons with HIV without LD and in persons with HIV and LD.

There is no cure for HIV. Therefore, persons living with HIV with or without LD are no longer just trying to survive day to day; rather, they are seeing the future of living with HIV as a chronic disease with debilitating longterm consequences. Direction for a future study needs to include better measures of biological/physiological factors that specifically assess current disease status. Specifically, work needs to focus on a specific liver disease that may significantly impact persons living with HIV such as the hepatitis $C$ virus or on specific symptoms that affect the individual most, such as depressive symptoms.

Depressive and mental symptoms had the strongest relationship to the other model measures suggesting that a focus for clinical intervention would be to more closely address these issues in persons living with HIV and LD. These findings also suggest that a more complete understanding of the symptom experience in persons living with HIV and $\mathrm{LD}$ is fundamental to achieve optimal patient outcomes. It may be that specific symptoms, such as depressive symptoms, need to be controlled for model testing. Future research should consider a symptom-specific tool to look for clusters of symptoms in both HIV and liver disease. This study also found that race, social support, and income were important covariates. Therefore, another direction for future research would be to assess differences between racial and socioeconomic status on HRQOL in persons with HIV and LD.

\section{Ethical Approval}

The study was approved by the Institutional Review Board of the University of Pittsburgh and the National Institutes of Health Office of Human Subjects Research.

\section{Acknowledgments}

The authors would like to acknowledge the patients who participated in the research study as well as the research staff of the parent study. The authors would also like to thank Elizabeth A. Schlenk, Judith T. Matthews, and Xiaoli Lu for their mentorship. The following are sources of funding: US Department of Health and Human Services; National Institutes of Health; National Institute of Nursing Research (W. A. Henderson 1ZIANR000018-03); Association of Nurses in AIDS Care and Sigma Theta Tau International; Epsilon Phi Chapter; Sigma Theta Tau International Research Award 2006; NIH clinical and translational fellowship (1 TL1 RR 024155-01); Parent study (NIH R01 NR04749; NIH 2R01 NR04749).

\section{References}

[1] P. Leger, M. Charles, P. Severe, C. Riviere, J. W. Pape, and D. W. Fitzgerald, "5-Year survival of patients with AIDS receiving antiretroviral therapy in Haiti," The New England Journal of Medicine, vol. 361, no. 8, pp. 828-829, 2009.

[2] C. L. Thio, "Hepatitis B and human immunodeficiency virus coinfection," Hepatology, vol. 49, supplement 5, pp. S138S145, 2009.

[3] A. Stewart, S. C. Carusone, K. To, N. Schaefer-McDaniel, M. Halman, and R. Grimes, "Causes of death in HIV patients and the evolution of an AIDS hospice: 1988-2008," AIDS Research and Treatment, vol. 2012, Article ID 390406, 7 pages, 2012.

[4] Antiretroviral Therapy Cohort Collaboration, "Causes of death in HIV-1-infected patients treated with antiretroviral therapy, 1996-2006: collaborative analysis of 13 HIV cohort studies," Clinical Infectious Diseases, vol. 50, pp. 1387-1396, 2010.

[5] R. Sanmartin, E. de Felipe, J. Tor et al., "Effect of liver fibrosis on long-term mortality in HIV/hepatitis C virus-coinfected individuals who are evaluated to receive interferon therapies in the highly active antiretroviral therapy era," AIDS Research and Human Retroviruses, vol. 28, no. 10, pp. 1235-1243, 2012.

[6] G. N. Ioannou, C. L. Bryson, N. S. Weiss, R. Miller, J. D. Scott, and E. J. Boyko, "The prevalence of cirrhosis and hepatocellular carcinoma in patients with HIV infection," Hepatology. In press.

[7] A. N. W. Maek, A. Avihingsanon, and P. J. Ohata, "Challenges in providing treatment and care for viral hepatitis among individuals Co-infected with HIV in resource-limited settings," AIDS Research and Treatment, vol. 2012, Article ID 948059, 10 pages, 2012. 
[8] A. P. Kourtis, M. Bulterys, D. J. Hu, and D. J. Jamieson, "HIVHBV coinfection - a global challenge," The New England Journal of Medicine, vol. 366, pp. 1749-1752, 2012.

[9] L. Highleyman, "HIV/HCV coinfection: a new era of treatment," BETA, vol. 23, pp. 30-47, 2011.

[10] V. Soriano, L. Martin-Carbonero, I. Maida, J. GarciaSamaniego, and M. Nuñez, "New paradigms in the management of HIV and hepatitis C virus coinfection," Current Opinion in Infectious Diseases, vol. 18, no. 6, pp. 550-560, 2005.

[11] C. J. Hoffmann and C. L. Thio, "Clinical implications of HIV and hepatitis B co-infection in Asia and Africa," Lancet Infectious Diseases, vol. 7, no. 6, pp. 402-409, 2007.

[12] H. Kovari and R. Weber, "Influence of antiretroviral therapy on liver disease," Current Opinion in HIV and AIDS, vol. 6, no. 4, pp. 272-277, 2011.

[13] L. P. Deng, X. E. Gui, Y. X. Zhang, S. C. Gao, and R. R. Yang, "Impact of human immunodeficiency virus infection on the course of hepatitis C virus infection: a meta-analysis," World Journal of Gastroenterology, vol. 15, no. 8, pp. 996-1003, 2009.

[14] E. Chak, A. H. Talal, K. E. Sherman, E. R. Schiff, and S. Saab, "Hepatitis C virus infection in USA: an estimate of true prevalence," Liver International, vol. 31, no. 8, pp. 1090-1101, 2011.

[15] G. Aragones, C. Alonso-Villaverde, C. Oliveras-Ferraros et al., "Infection with HIV and HCV enhances the release of fatty acid synthase into circulation: evidence for a novel indicator of viral infection," BMC Gastroenterol, vol. 10, p. 92, 2010.

[16] G. R. Foster, R. D. Goldin, and H. C. Thomas, "Chronic hepatitis $\mathrm{C}$ virus infection causes a significant reduction in quality of life in the absence of cirrhosis," Hepatology, vol. 27, pp. 209-212, 1998.

[17] USDHHS, “Healthy People 2020," United States Department of Health and Human Services, 2010.

[18] I. B. Wilson and P. D. Cleary, "Linking clinical variables with health-related quality of life: a conceptual model of patient outcomes," Journal of the American Medical Association, vol. 273, no. 1, pp. 59-65, 1995.

[19] D. Chadwick, "Measuring antiepileptic therapies: the patient vs the physician viewpoint," Neurology, vol. 44, no. 11, supplement 8, pp. S24-S28, 1994.

[20] D. Horstkotte, "Quality of life after heart valve replacement," in Quality of Life after Open Heart Surgery, P. J. Walter, Ed., pp. 101-104, Kluwer Academic, Boston, Mass, USA, 1992.

[21] M. E. Hyland and C. A. Kenyon, "A measure of positive healthrelated quality of life: the Satisfaction with Illness Scale," Psychological Reports, vol. 71, pp. 1137-1138, 1992.

[22] R. M. Kaplan and J. P. Anderson, "The general health policy model: an integrated approach," in Quality of Life and Pharmacoeconomics in Clinical Trials, B. Spilker, Ed., pp. 309-322, Lippincott-Raven, Philadelphia, Pa, USA, 2 edition, 1996.

[23] S. Levy and L. Guttman, "On the multivariate structure of wellbeing," Social Indicators Research, vol. 2, no. 3, pp. 361388, 1975.

[24] M. A. Testa and D. C. Simonson, "Assessment of quality-of-life outcomes," The New England Journal of Medicine, vol. 334, no. 13, pp. 835-840, 1996.

[25] J. E. Ware, "Methodology in behavioral and psychosocial cancer research. Conceptualizing disease impact and treatment outcomes," in Cancer, vol. 53, pp. 2316-2326, 1984.

[26] A. W. Wu, D. A. Revicki, D. Jacobson, and F. E. Malitz, "Evidence for reliability, validity and usefulness of the Medical
Outcomes Study HIV Health Survey (MOS-HIV)," in Quality of Life Research, vol. 6, pp. 481-493, 1997.

[27] J. A. Erlen, "Adherence to protease inhibitors (R01 NR04749) funded by the National Institute of Nursing Research," 1998.

[28] E. Diener, R. A. Emmons, R. J. Larsen, and S. Griffin, "The satisfaction with life scale," Journal of Personality Assessment, vol. 49, pp. 71-75, 1985.

[29] S. Cohen, R. Mermelstein, T. Kamarck, and H. Hoberman, "Measuring the functional components of social support," in Social Support: Theory, Research and Application, I. G. Sarason and B. R. Sarason, Eds., Martinus Nijhoff, The Hague, Holland, 1985.

[30] P. M. Bentler and D. G. Bonett, EQS Structural Equations Program Manual, Multivariate Software, Encino, Calif, USA, 2002.

[31] D. L. Jackson, "Revisiting sample size and number of parameter estimates: some support for the N : q hypothesis," Structural Equation Modeling, vol. 10, pp. 128-141, 2003.

[32] N. N. Anandan, B. Braveman, G. Kielhofner, and K. Forsyth, "Impairments and perceived competence in persons living with HIV/AIDS," Work, vol. 27, no. 3, pp. 255-266, 2006.

[33] S. Crystal, J. A. Fleishman, R. D. Hays, M. F. Shapiro, and S. A. Bozzette, "Physical and role functioning among persons with HIV: results from a nationally representative survey," Medical Care, vol. 38, no. 12, pp. 1210-1223, 2000.

[34] W. A. Henderson, J. M. Fall-Dickson, E. A. Schlenk, K. H. Kim, J. T. Matthews, and J. A. Erlen, "Effects of liver disease on the well-being of persons living with HIV," Journal of the Association of Nurses in AIDS Care, vol. 19, no. 5, pp. 368-374, 2008.

[35] W. A. Henderson, E. A. Schlenk, K. H. Kim et al., "Validation of the MOS-HIV as a measure of health-related quality of life in persons living with HIV and liver disease," AIDS Care, vol. 22, no. 4, pp. 483-490, 2010.

[36] A. L. Hudson, K. A. Lee, and C. J. Portillo, "Symptom experience and functional status among HIV-infected women," AIDS Care, vol. 15, no. 4, pp. 483-492, 2003.

[37] A. Hughes, "Symptom management in HIV-infected patients," Journal of the Association of Nurses in AIDS Care, vol. 15, no. 5, supplement, pp. 7S-13S, 2004.

[38] R. Murri, M. Fantoni, C. Del Borgo et al., "Determinants of health-related quality of life in HIV-infected patients," AIDS Care, vol. 15, no. 4, pp. 581-590, 2003.

[39] N. A. Phaladze, S. Human, S. B. Dlamini et al., "Quality of life and the concept of "Living well" with HIV/AIDS in subSaharan Africa," Journal of Nursing Scholarship, vol. 37, no. 2, pp. 120-126, 2005.

[40] K. H. Sousa and O. M. Kwok, "Putting Wilson and Cleary to the test: analysis of a HRQOL conceptual model using structural equation modeling," Quality of Life Research, vol. 15, no. 4, pp. 725-737, 2006.

[41] D. J. Vidrine, B. C. Amick, E. R. Gritz, and R. C. Arduino, "Assessing a conceptual framework of health-related quality of life in a HIV/AIDS population," Quality of Life Research, vol. 14, no. 4, pp. 923-933, 2005.

[42] J. G. Voss, "Predictors and correlates of fatigue in HIV/AIDS," Journal of Pain and Symptom Management, vol. 29, no. 2, pp. 173-184, 2005.

[43] R. Dray-Spira and F. Lert, "Social health inequalities during the course of chronic HIV disease in the era of highly active antiretroviral therapy," AIDS, vol. 17, no. 3, pp. 283-290, 2003. 
[44] W. McFarland, S. Chen, L. Hsu, S. Schwarcz, and M. Katz, "Low socioeconomic status is associated with a higher rate of death in the era of highly active antiretroviral therapy, San Francisco," Journal of Acquired Immune Deficiency Syndromes, vol. 33, no. 1, pp. 96-103, 2003.

[45] B. D. Rapkin and C. E. Schwartz, "Toward a theoretical model of quality-of-life appraisal: implications of findings from studies of response shift," Health and Quality of Life Outcomes, vol. 2, article 14, 2004. 


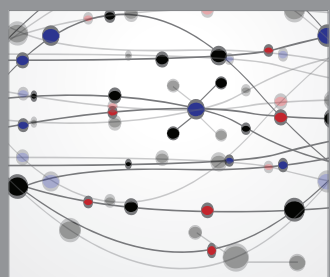

The Scientific World Journal
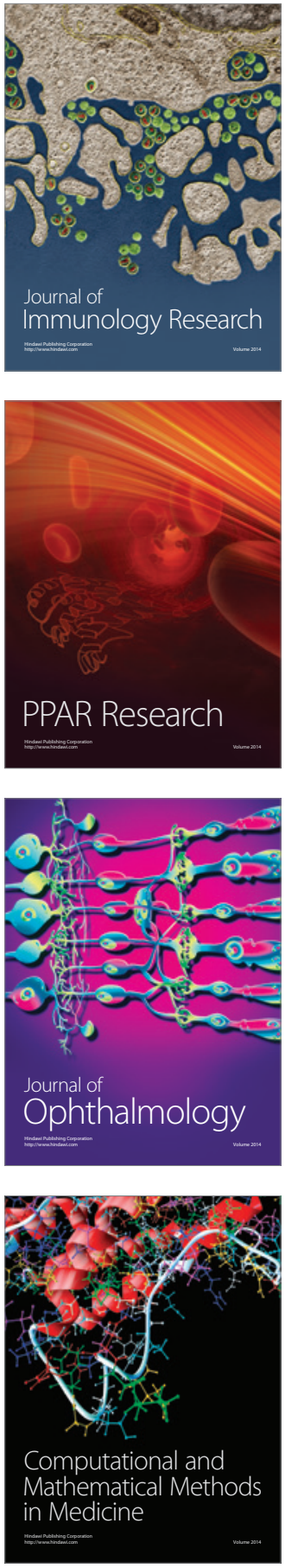

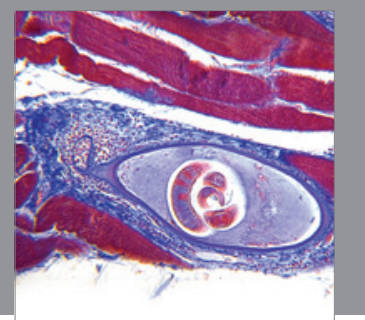

Gastroenterology

Research and Practice
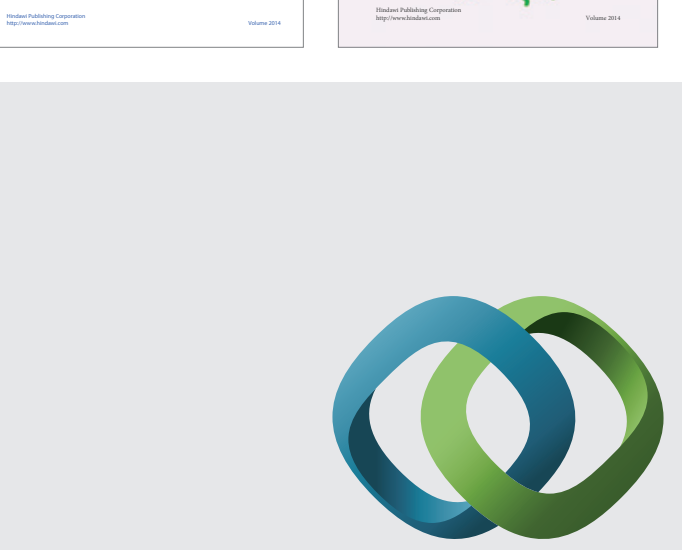

\section{Hindawi}

Submit your manuscripts at

http://www.hindawi.com
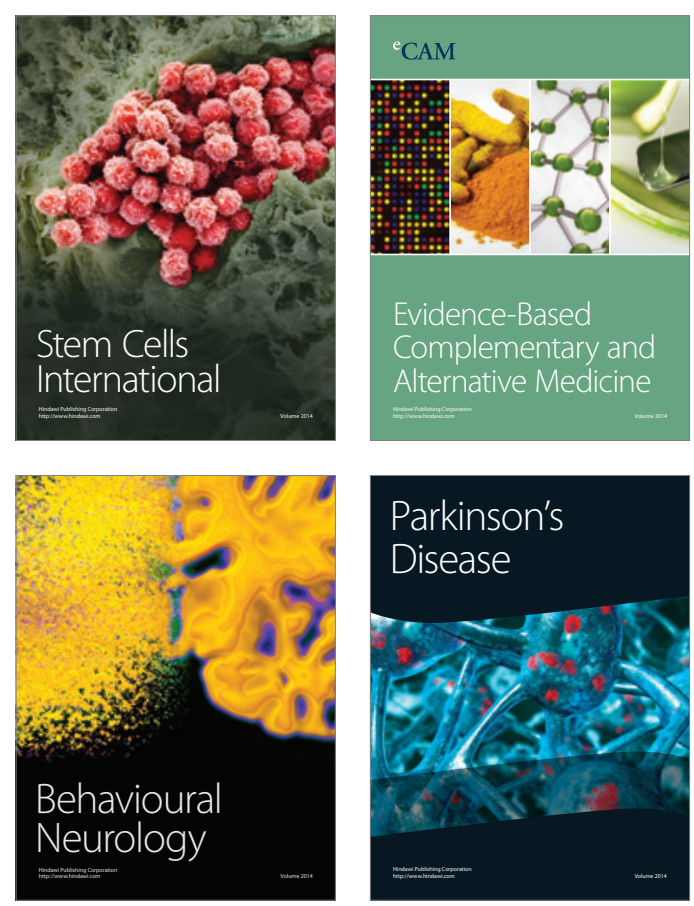

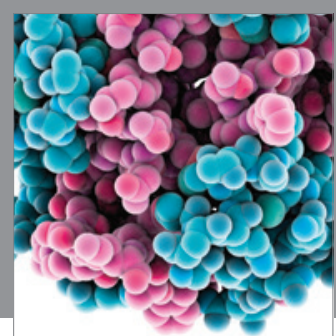

Journal of
Diabetes Research

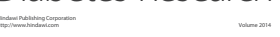

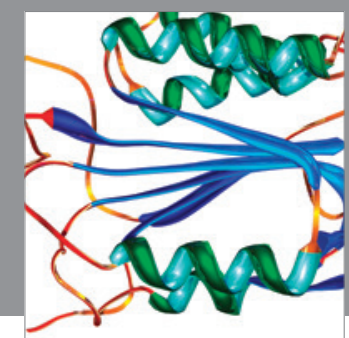

Disease Markers
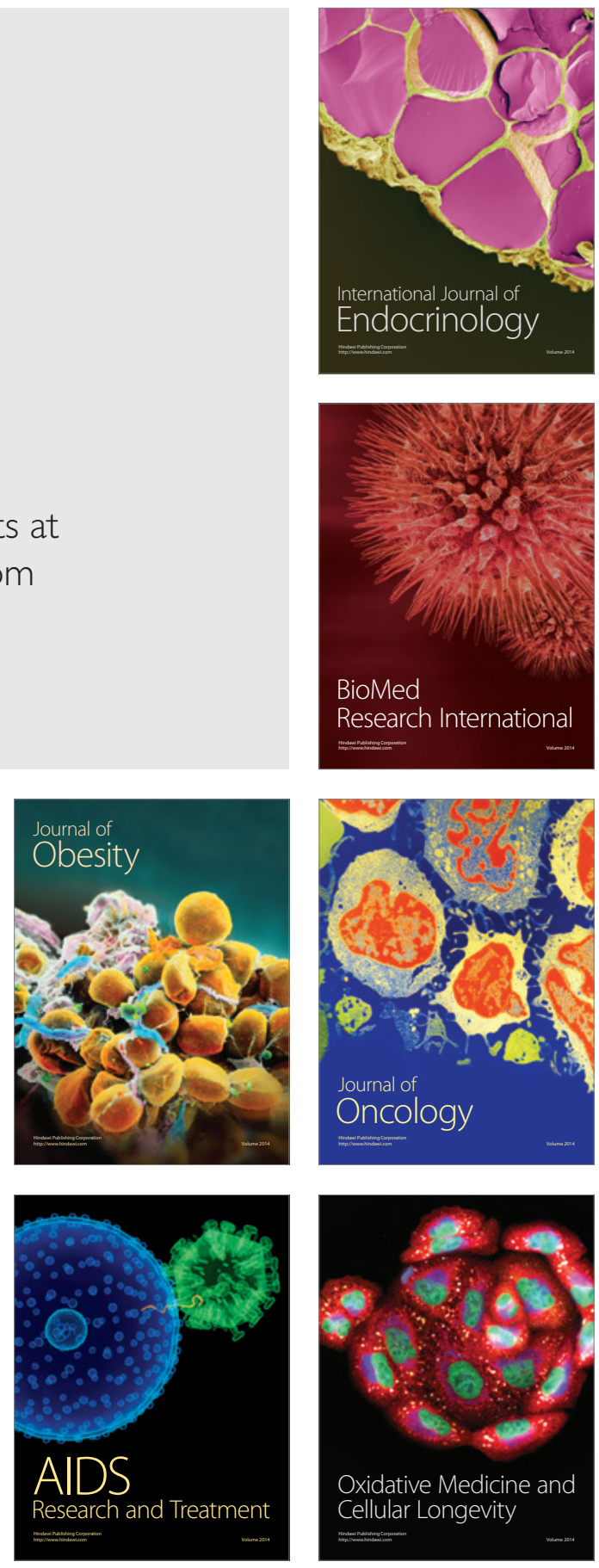